\title{
Uso da informação na gestão de hospitais públicos
}

\author{
Information use in public hospital management
}

Abstract Thisstudy investigates managerial perceptions of the use of information in health management and planning in 24 Public Hospitals in the São Paulo M etropolitan Region, analyzing its usefulness for the decision-making process. In addition, some characteristics of the existing information system are studied. The findings show that ampleamounts of information and data are available in the hospitals covered by this study, despite some gaps, and that managers do not know about the existing data and do not use this information to guide hospital management.

Key words Hospital indicators, Health indicator use, Hospital management, Hospital information systems, M anagerial epidemiology
Resumo Analisa-se a utilização da informação para a tomada de decisão, segundo a visão dos diretores e dos responsáveis pela área de informações dos 24 hospitais públicos estaduais da administração direta da Região M etropolitana de São Paulo, assim como alguns aspectos rel ativos aos sistemas de informação existentes. Os resultados mostram que esses hospitais produzem um considerável volume de dados, embora com lacunas importantes, e que muitos gestores desconhecem a existência de tais informações ou não as utilizam adequadamente para subsidiar a gestão hospitalar.

Palavras-chave Indicadores hospitalares, Uso de indicadores de saúde, Gestão hospitalar, Sistemas de informação hospitalar, Epidemiologia gerencial 


\section{Introdução}

No contexto atual, intensifica-se a utilização de indicadores e informações para comparar as organizações de saúde, visando levá-las a níveis de superioridade evantagem competitiva, por meio de referências (benchmarks) de processos, práticas ou medidas de desempenho ${ }^{1}$.

Cresce também a exigência para que os serviços de saúde, tanto privados quanto da área pública, organizem-se de modo a responder às necessidades das pessoas e ofereçam um cuidado efetivo e humanizado, provendo todas as informações que o usuário necessita².

Pesquisadores e profissionais que atuam na área de Administração em Saúde têm sido unânimes em relatar tanto a complexidade envolvida na tarefa de eleger os sistemas de avaliação e os indicadores de desempenho institucional mais adequados para apoiar a gestão dos hospitais, como a necessidade de serem estudadas as características e os fatores determinantes do uso destes instrumentos pelos administradores ${ }^{3}$.

Nesse processo, identifica-se um maior interesse pelo uso da metodologia epidemiológica na avaliação desistemas, serviços, programas eações de saúde, envolvendo: avaliação da qualidade; avaliação tecnológica - eficácia, segurança e efetividade; e avaliação econômica (eficiência) - custo-efetividade, custo-utilidade, custo-benefício. 0 equacionamento dessas complexas questões requer a utilização de conceitos e ferramentas da Epidemiologia Gerencial ${ }^{4}$, assim como de diversas outras disciplinas, como a economia e a administração $0^{5}$, para produzir as informações técnicas e científicas (evidências) essenciais na definição das necessidades e avaliação dos resultados, a partir da perspectiva populacional.

Dobrow et al. ${ }^{6}$ sugerem ser necessário fazer uma distinção entre o "impacto das evidências" nos resultados das organizações e a simples "utilização das evidências" no processo de tomada de decisão. Esseéum dos principais desafios para 0 entendimento do "decision-making", já que decisões baseadas em uma mesma evidência científica podem apresentar resultados diversos quando utilizadas em distintos contextos. Essa complexidade decorre do fato de que tanto 0 conhecimento científico produzido pelas pesquisas admite distintas interpretações, quanto 0 "processo de tomada de decisão baseado em evidências" (evidence-based decision-making) é afetado por múlti plas outras variáveis além da própria evidência. Assim, a utilização e 0 impacto das evidências na tomada de decisão dependerão de como elas são selecionadas, interpretadas e valorizadas pelas diversas partes envolvidas, refletindo valores pessoais e interesses dos tomadores de decisão, bem como seus juízos sobre a qualidade das fontes utilizadas.

\section{Desempenho equalidade}

0 projeto Performance assessment tool for quality improvement in hospitals - PATH identificou seis dimensões interdependentes para a avaliação do desempenho hospitalar: efetividade clínica, segurança, foco no paciente ( patient centredness), eficiência na produção, orientação do staff e governança receptiva ${ }^{7}$. Por meio de uma extensa revisão da literatura e da construção de consenso entre especialistas, foi selecionado um set decem indicadores edesenvolvidososinstrumentos para o benchmarking dos resultados entre os hospitais participantes, entreesses um painel demonitoramento (balanced dashboard).

Essas e outras experiências que estão sen do conduzidas em muitos países mostram que a disponibilidade de sistemas adequados de informações epidemiológicas e administrativas é considerada um fator crítico de sucesso para o gerenciamento ético do cuidado em saúde, devendo permitir não somente a avaliação econômica, como também a da qualidade dos serviços prestados 8 .

Por outro lado, como apontam Turpin et al. ${ }^{9}$, os indicadores de desempenho não são medidas diretas da qualidade e sim "flags to alert users to possible opportunities for improvement in processes and outcomes". Ou seja, rigorosamente os indicadores de desempenho mensuram apenas os aspectos do cuidado em saúde que os tomadores de decisão pretendem controlar, embora, freqüentemente, eles estejam associados à qualidade dos resultados dos serviços de saúde.

Sabe-se que qualidade é um termo abstrato que vem sendo definido ao longo do tempo de distintas formas decorrentes das necessidades das organizações e dos objetivos dos avaliadores. As definições mais recentes tendem a complementar as mais antigas, podendo esse termo ser entendido como excelência, como valor, como conformidade a critérios definidos e como satisfação dos usuários dos serviços de saúde.

$N$ ão existe consenso na literatura quanto à definição e classificação dos indicadores de qualidade, sendo possível identificar uma tendência de se fazer uma distinção entre as dimensões estrutura, processo e resultados da qualidade, se- 
guindo a clássica formulação de Donabedian ${ }^{10}$. 0 s indicadores de resultado referentes à dimensão "satisfação do usuário" deveriam, em tese, refletir as outras dimensões, ou seja, a satisfação poderia ser entendida como uma variável independente, determinada pelas dimensões: acesso, efetividade do cuidado e custo. Por sua vez, a avaliação da efetividade do cuidado requer 0 emprego de indicadores objetivos, tais como a duração do tratamento, ocorrência de recidivas e efeitos adversos de medicamentos. É necessário, portanto, construir indicadores para medir, ao mesmo tempo, o atendimento às necessidades do usuário, definidas tecnicamente, e as suas preferências.

\section{Seleção de indicadores e disponibilização da informação}

Três questões são importantes para o desenvolvimento e aplicação de indicadores: qual a perspectiva que 0 indicador pretende refletir; quais aspectos do cuidado em saúde serão mensurados/avaliados; quais são as evidências científicas disponíveis.

A seleção do conjunto deindicadores edo seu nível de desagregação pode variar em função das necessidades específicas e prioridades de cada instituição, da disponibilidade de sistemas de informação e fontes de dados e dos recursos alocados nessa atividade. Portanto, é uma tarefa complexa selecionar qual informação énecessária ao gestor dos serviços de saúde, bem como avaliar a sua contribuiç̧ão para decisões mais acertadas, considerando as distintas perspectivas dos indivíduos, grupos ou entidades que têm algum interesse ou influência em uma organização específica (stakeholders).

Para tanto, é essencial existir uma interação entre os que tomam decisões e os que organizam e gerenciam os sistemas de informação, com a finalidade de definir os dados e informações relevantes e a forma mais adequada, em cada organização, para coletar, processar, armazenar e disseminar a informação necessária para tomada de decisão, coordenação, controle, análise e visualizaçãa ${ }^{11}$. Ressalte-se também que a quantidade e a forma de apresentação da informação para os que tomam as decisões são requisitos importantes.

O utro aspecto relevante é que uma mesma informação pode ser usada de diversas formas, em diversos momentos e por diversas pessoas durante o processo decisório, ocorrendo intera- ções complexas entre a capacidade cognitiva dos usuários, a natureza e o formato da informação. Sendo processadores de informação, os tomadores de decisão atuam de acordo com o volume, qualidade e acessibilidade dos dados disponíveis, bem como de sua capacidade para analisá-los. 0 modelo conceitual sobre a capacidade de processar informações dos indivíduos, desenvolvido por Schroeder e colaboradores, mostra que quando a quantidade de informação disponível para um gestor aumenta, cresce o uso dessa informação até chegar a um ponto de utilidade máxima, a partir do qual qualquer incremento adicional da quantidade de informação resulta em menor utilidade para o gestor, ou seja, existe um limite da capacidade cognitiva de cada indivíduo para processar informações, além do qual ocorre a sobrecarga de informação (information overload). Os estudos mostram ainda a vantagem comparativa em analisar informações em equipe, pois a capacidade de processamento da informação do grupo é maior que a soma das capacidades individuais de seus integrantes trabalhando isoladamente ${ }^{12}$.

Existem diversos sistemas relacionando as estratégias organizacionais com indicadores de desempenho e de gestão com vistas a facilitar a utilização da informação pelos tomadores de decisão. Estes sistemas fornecem uma rápida, porém abrangente, visão dos indicadores de desempenho vis-à-vis os objetivos de qualidade, operacionais e financeiros ${ }^{13}$. 0 uso de uma dessas ferramentas, o Balanced Scorecard - BSC, vem se ampliando na área de saúde e diversas experiências têm sido divulgadas, revelando tanto 0 potencial dessa metodologia, como as dificuldades para a sua implementação nas organizações de saúde ${ }^{14}$.

O BSC promove o alinhamento da organização, dos indivíduos e das iniciativas interdepartamentais, possibilitando que os esforços sejam dirigidos para as áreas que necessitem de maior atenção, utilizando indicadores classificados segundo quatro categorias básicas: finanças; clientes; processos internos; aprendizagem e crescimento. Uma das grandes dificuldades para operacional izar esse sistema éa obten ção dos dados, pois envolve a superação de problemas relativos aos sistemas de produção das informações nas empresas, como a impossibilidade de se obter dados relativos a períodos de tempo menores ${ }^{15}$.

Pesquisas e relatos de experiências brasileiras também têm mostrado a utilidade da construção de painéis de controle para avaliar a performance das ações desenvolvidas nos distintos ní- 
veis deatenção à saúde, inclusivenas instituições públicas ${ }^{16}$. Uma dessas experiências, o “Painel de monitoramento das condições de vida e saúde da população e da situação dos serviços de saúde", da Secretaria M unicipal da Saúde de São Pau$10^{17}$, disponibiliza um panorama sintético para 0 acompanhamento da gestão do sistema municipal de saúde, em suas várias instâncias de organização, e para subsidiar os gestores no acompanhamento e avaliação das ações sob sua responsabilidade, constituindo ainda um instrumento de democratização da informação sobre os resultados alcançados pela Secretaria.

Para cada nível de gestão, devem ser selecionados indicadores relativos aos processos sob sua respectiva responsabilidade, sendo estes indicadores disponibilizados em intervalos detempos adequados aos processos de tomada de decisões de cada um desses níveis. Este ajuste entre as estratégias de negócio, tecnologias de informação e estruturas internas da empresa não é um resultado simples de ser obtido, mas sim um processo dinâmico econtínuo. 0 impacto da tecnologia dependerá da capacidade da organização em explorá-la de forma contínua e o seu sucesso não está apenas relacionado com o hardware e o software utilizados, ou ainda com metodologias de desenvolvimento, mas com o alinhamento da TI à estratégia e às características da empresa e de sua estrutura organizacional ${ }^{18}$.

Existem diversos estudos mostrando os benefícios da TI para a melhora da eficácia, segurança e eficiência dos serviços de saúde ${ }^{19}$, embora a incorporação dessas aplicações varie significativamente de acordo com o porte e os financiadores dos hospitais, entre outros fatores. Por outro lado, não existe ainda clareza sobre quais tipos de soluções podem redundar em melhor qualidade do cuidado emenor custo e, também, sobrequais seriam as fontes de financiamento da incorporação de TI nas organizações de saúde ${ }^{20}$.

\section{U so da informação para a tomada de decisão}

A profissionalização da gestão na área de saúde deixou de ser uma vantagem competitiva ou um diferencial da instituição, tornando-se um determinante básico, como na maioria dos outros setores da economia. Porém, a idéia degerir baseando-se em informação ainda não foi assimilada completamente nesse setor. Impõe-se, portanto, perguntar: com tantos recursos disponíveis nesta nossa "era da informação", como explicar que a falta de comunicação edisseminação deinforma- ção ainda seja uma barreira para um adequado processo de tomada de decisão? Por que os gestores enfrentam tanta dificuldade para partilhar seus conhecimentos? Pareceser bastante claro queeste não é um problema de TI, e sim humano.

Em um modelo perfeito da partilha do conhecimento, os administradores são valorizados não porque sabem mais do que os seus subordinados, mas porque conseguem rapidamente lhes comunicar o que sabem e, ainda, porque conseguem que eles façam o mesmo entresi. Entretanto, nas organizações humanas, existe sempre um movimento complexo de disputa, com focos de instabilidade, riscos de conflitos einteresses múltiplos disputando hegemonia em situações estratégicas, o que potencializa uma leitura restrita da expressão clássica: "informação é poder"21. Assim, habitualmente, os líderes das equipes guardam as informações, partilhando-as apenas quando é imprescindível.

Um conjunto de inibidores pessoais e organizacionais tem sido apontado para explicar a falta de compartilhamento das informações nas empresas: crença generalizada na idéia de que "se as pessoas sabem algo que os outros não sabem, possuem vantagem competitiva sobre eles"; insegurança que muitos têm quanto ao valor do seu próprio conhecimento e a falta de confiança nas outras pessoas. Freqüentemente, também, não se partilha a informação por medo de conseqüências negativas, o que torna crucial eliminar as barreiras ao fluxo livre de idéias e criar uma cultura de disseminação do conhecimento ${ }^{22}$.

Um outro obstáculo para a utilização de indicadores no processo decisório éa falta de confiança dos gestores na fidedignidade dos dados. No entanto, em que pese a relevância do aprimoramento e monitoramento constantes da qualidade dos indicadores, a principal forma de aprimorar as bases de dados é a disseminação e a utilização da informação. Finalmente, a utilização da informação para a tomada de decisão depende da forma de apresentação da informação para os gestores e da cultura das organizações. Registre-se, porém, a carência de profissionais capacitados para analisar os dados e informações hospitalares e implantar modelos adequados de disponibilização dos mesmos aos diversos interessados ${ }^{23}$.

\section{Indicadores hospitalares}

Um dos principais problemas enfrentados para a modelagem dos sistemas de informação hos- 
pitalares éa definição e a forma de medir os produtos ${ }^{24}$. Em geral, o produto hospitalar é analisado pelo conjunto de serviços disponíveis (service mix) ou pelo conjunto de pacientes (case mix). Uma das limitações destas abordagens é enfocar aspectos gerais da produção hospitalar, não considerando o perfil dos pacientes, que são a razão da existência dos serviços de saúde ${ }^{25}$.

0 aumento nos custos dos cuidados médicos nos Estados Unidos da América, nas décadas de 60 e de 70, em que predominavam os sistemas de pós-pagamento (per diem compensation system) e de pagamento por procedimento (fee forservice) provocou a reestruturação das formas de financiamento da assistência à saúde, implantando-se o Prospective Payment System - PPS, cujos principais objetivos foram reduzir a taxa depermanência hospitalar eminimizar a realização de procedimentos desnecessários. Este sistema definia uma quantia fixa a ser paga para cada alta hospitalar de casos agudos.

A implantação do PPS exigiu a criação deuma base de dados para definir as quantias que seriam pagas por paciente atendido. A U niversidade de Yale desenvolveu, no final da década de 60, 0 "Diagnosis Related Groups - DRGs" (Grupos de Diagnósticos Homogêneos - GDH), um sistema que relaciona o case mix dos hospitais (relação proporcional entre os diferentes tipos de pacientes atendidos em um determinado serviço) com o tipo de tecnologia utilizada no cuidado médico e seus custos correspondentes.

Os grupos de diagnósticos homogêneos definem como produtos hospitalares os pacientes egressos, agrupados de acordo com o perfil de recursos recebidos durantea internação. Esteconceito parte do pressuposto de que grupos de doentes tenham atributos demográficos, diagnósticoseterapêuticos comuns, que determinariam as condutas médicas adotadas. Assim, o tempo de internação seria uma variável dependente de algumas variáveis independentes, tais como: diagnóstico principal, procedimento principal, presença de complicações importantes ou diagnósticos associados, outros procedimentos operatórios, idade do paciente e condição de saída ${ }^{26}$.

Pesquisas já demonstraram o impacto do uso de DRG para análise financeira, verificando-se uma redução considerável do custo por alta e decréscimos importantes na média de permanên$\mathrm{cia}^{27}$. Por outro lado, embora o DRG tenha sido desenvolvido para analisar custos e efetuar pagamentos, estudos têm mostrado a utilidade do DRG para a construção de indicadores de desempenho hospitalar, como por exemplo, a dis- tribuição das taxas de reinternação de pacientes, segundo os códigos do DRG ${ }^{28}$.

Diversas versões de DRG vêm sendo desenvolvidas e atualizadas regularmente, inclusive para classificar pacientes atendidos em ambulatórios e serviços de cuidados prolongados, sendo atualmente utilizado como base para alocação de recursos e pagamentos em vários países da Europa e na Austrália29.

$M$ ais recentemente, observa-sea tendência da incorporação de soluções de TI para apoiar as práticas de melhoria da qualidade do cuidado (Quality Improvement - QI) e a segurança do paciente ${ }^{30,31}$. Com esse propósito, a Agency for Healthcare Research and Quality - AHRQ vem disponibilizando gratuitamenteum conjunto de softwares visando facilitar a comparação do desempenho dos hospitais americanos ${ }^{32}$. De modo geral, considera-sequeessa iniciativa tenha apresentado resultados positivos, embora os resultados da implantação dessas soluções em cada hospital dependam da capacidadee eficiência dos sistemas de informação existentes, da alocação derecursos financeiros para as atividades deQ I e da integração dos sistemas clínicos ${ }^{33}$. A divulgação ampla dos dados sobre o desempenho dos hospitais americanos, com reflexo na imagem desses serviços junto aos usuários, tem sido também apontada como um fator de melhoria da qualidade $\mathrm{e}^{34}$.

No Brasil, a principal base de dados para o estudo das internações hospitalares éo SI H -SUS, modelado no final da década de 70 e implantado em todo o país entre 1983 e 1984. Segundo N oronha $a^{35}$, o SIH pode ser considerado "avançado para o momento em que foi criado"; no entanto, a falta de revisão do sistema de classificação e de incorporação dos avanços tecnológicos tornou o sistema desatualizado diante de outros que foram desenvolvidos eaprimorados ao longo desse período. Existem restrições em seu uso para a avaliação da morbidade hospitalar, podendo-se, entre outras, citar que as informações referem-se ao evento "internação" e não às "pessoas internadas", o que impede a construção de coeficientes.

Somente na década de 90 , observa-se um uso mais abrangente do $\mathrm{SIH}$, o que talvez tenha sido viabilizado pela maior facilidade de acesso ao banco de dados. Bittencourt et al. ${ }^{36}$ ressaltam que os maiores problemas nessa base de dados estão relacionados à qualidade do diagnóstico da internação, o que leva os pesquisadores a classificarem os motivos de internação em capítulos ou em grupos de causa da CID, em vez da categoria específica. 
Zanetta ${ }^{37}$ considera que 0 sistema DRG pode servir de base para uma revisão da tabela de procedimentos e remuneração das internações do SUS queeliminedistorções ediferencieintervenções hospitalares segundo gravidade e complexidade dos casos e propõe a revisão do sistema de classificação da AlH para que este possa diferenciar os casos segundo gravidade e complexidade da atenção, incorporando conceitos de co-morbidades, complicações e consumo de recursos hospitalares na sua conformação.

Outro aspecto que limita a comparação de indicadores entre hospitais é o uso de definições distintas, o que ocorre inclusive entre as diversas áreas de um mesmo serviço, sendo um exemplo emblemático a dificuldade que se encontra habitualmente para conhecer, com exatidão, o número deleitos existentes em um hospital, o quecompromete a fidedignidade dos indicadores hospitalares. A propósito, o M inistério da Saúde, por meio da Portaria no 312, de 02 de maio de 2002, estabeleceu uma padronização da nomenclatura do censo hospitalar, o que pode contribuir para minimizar esse problema ${ }^{38}$.

\section{Objetivos}

O objetivo do estudo é conhecer e analisar a utilização de indicadores e informações no planejamento e na gestão dos hospitais públicos da Região M etropolitana de São Paulo, segundo a visão dos diretores hospitalares e dos responsáveis pela área de informações/SAM E. Procurousetambém conhecer o perfil da equipe profissional responsável pela análise dos dados e elaboração de relatórios gerenciais, assim como algumas características dos sistemas de informação existentes.

\section{Metodologia}

A pesquisa foi dirigida para os 24 hospitais próprios vinculados à Coordenadoria de Saúde da Região M etropolitana da Grande São Paulo da SES de São Paulo (atualmente vinculados à Coordenadoria de Serviços de Saúde - CSS), utilizando-se dois tipos de questionários semi-estruturados, aplicados por dois profissionais especialistas em Administração Hospitalar, no período de fevereiro a maio do ano 2000.

No roteiro para os diretores, procurou-se conhecer os dados, indicadores e informações disponíveis, bem como sua utilização no plane- jamento e na gestão do hospital. Interessava também conhecer a maneira como os diretores selecionam estas informações técnicas e se as comparam com as de outros hospitais de referência. Foram solicitados exemplos do uso de indicadores, visando discriminar no discurso dos entrevistados "a intenção" do diretor em usar indicadores da "prática regular de uso" no seu cotidiano de trabalho. N esta direção, encaixaram-se questões relativas aos mecanismos de divulgação dos dados e das informações.

Nos quesitos referentes às características do sistema de informação existente, buscou-se não apenas conhecer a situação destes sistemas nos hospitais estudados (grau de informatização, definição de fluxos, profissionais envolvidos, etc.), como investigar o grau de interesse e dedicação dos diretores ao assunto. Eles foram também instados a expressar suas avaliações sobre a qualidade dos sistemas de informação existentes em seus respectivos hospitais.

As questões sobre o perfil de morbidade dos pacientes e os instrumentos que a diretoria do hospital usa para conhecê-lo focaram outra carência esperada na realidade da administração em saúde brasileira, que é a ausência de uma visão epidemiológica no planejamento egestão dos hospitais.

Finalmente, exploraram-se outras formas consideradas imprescindíveis no manejo das informações, que são a análise de séries históricas dos dados e indicadores de um mesmo serviço, já que esta é uma das poucas maneiras viáveis, em nossa realidade atual, para avaliar a performance. Outro método preconizado para avaliar os serviços, o benchmarking, foi incluído no roteiro, pedindo-se aos diretores para comparar o seu hospital com outros hospitais públicos pertencentes à administração direta, assim como hospitais de outra natureza jurídica.

Também foi solicitada a opinião dos diretoressobreum "elenco ideal" deindicadores, visando descobrir o grau de conhecimento dos mesmos sobre as principais propostas relativas à construção e ao uso dos indicadores hospitalares existentes na literatura. Os quesitos finais buscaram conhecer os fluxos das informações entre o hospital e os órgãos superiores da SES e saber a opinião dos diretores sobre os mesmos.

0 roteiro de entrevista com os responsáveis pelo SAM E/Setor de Informações era mais simplificado e continha al gumas questões semel hantes ao dos diretores, no sentido de permitir confrontar as duas visões sobre o mesmo quesito.

A análise do material transcrito procurou 
identificar nos relatos dos entrevistados as idéias relevantes, semelhantes, complementares ou opostas em relação a cada um dos quesitos selecionados. Em alguns hospitais, não foi possível entrevistar o diretor e/ou o responsável pela área deinformação, sendo queao final da fase de campo foi possível obter algum tipo de informação de 21 hospitais. Não foram incluídos os hospitais estaduais administrados pelas O rganizações Sociais de Saúde.

\section{Resultados ediscussão}

As respostas dos diretores hospitalares referentes ao quesito "indicadores hospitalares disponíveis para as atividades de planejamento e gestão" foram, em sua maior parte, referências aos indicadores denominados "clássicos", ou seja, indicadores consagrados pelo uso, tais como: taxa de ocupação, média de permanência e taxa de mortalidade institucional, entre outros. Foram mencionados, embora com menor freqüência, indicadores de produção, de qualidade, indicadores de infecção hospitalar e indicadores financeiros.

Já os responsáveis pela área de informação, em relação ao mesmo item acima citado, relataram a disponibilidade dos indicadores denominados "clássicos", indicadores de produção, indicadores solicitados pelo nível central da Secretaria, indicadores financeiros e "indicadores de hotelaria". Ressalte-se que, em apenas cinco dos hospitais entrevistados, as respostas do diretor do hospital e a do responsável pelo SAME a este quesito foram similares.

Quanto ao item "organização do sistema de fornecimento de dados, indicadores e relatórios", quase todos os diretores entrevistados afirmaram que o fluxo da informação provinha do SAM E. Porém, em al guns hospitais, o diretor do SAM E relatou dispor de vários indicadores que em nenhum momento foram mencionados pelo diretor do hospital evice-versa. Em al guns casos, apesar de os diretores dos hospitais afirmarem que recebiam as informações técnicas do SAME, eles mesmos calculavam uma série de indicadores que o próprio setor desconhecia; e, ainda, houve relatos da situação contrária, em que os indicadores produzidos pelo SAM E eram desconhecidos para a diretoria do hospital.

Em alguns hospitais, os diretores recebiam de modo desarticulado dados de diversos setores, sendo citados como exemplos a comissão de infecção hospitalar ea área de faturamento. Esta parece ser uma característica freqüente nos hos- pitais estudados e revela as dificuldades de comunicação na equipe e a falta de integração dos sistemas de informação.

Quando à "seleção dos indicadores", os diretores dos hospitais, em geral, concordam queesta não é realizada de acordo com as estratégias da instituição, sendo escolhidos os indicadores consagrados pelo uso, ou ainda de acordo com o queé solicitado pelo nível central da Secretaria da Saúde, ou seja, seguem padrões estipulados, sem os customizar às necessidades do gestor da instituição. Ressalte-se, no entanto, que al guns poucos diretores afirmaram selecionar seus indicadores baseando-se na literatura científica e/ou com base em discussões internas e experiências anteriores.

As considerações dos diretores hospitalares quanto "à avaliação e ao aprimoramento de seus processos de sel eção eutilização de indicadores a partir do conhecimento das informações de outros hospitais" mostram que, embora boa parte compare seus indicadores com os de hospitais similares, o fazem de modo informal e ocasional, em geral quando 0 administrador vai a algum simpósio ou congresso. Alguns diretores que referiram não realizar esta comparação apontam como principal entrave 0 acesso restrito às informações de outros hospitais.

Quando se solicitou um exemplo de uso bemsucedido de indicadores para o planejamento estratégico e para a gestão, quase todos os diretores hospitalares declararam queutilizam indicadores para estes fins, apesar de não terem diferenciado os indicadores para planejamento estratégico, dos indicadores para gestão, exemplificando como segue: "indicadores para controle de estoque de materiais; indicadores deinfecção hospitalar; taxa de cesáreas; uso de indicadores de produção para justificar abertura ou fechamento de um serviço, ou ainda, ajuste do quadro de funcionários e uso de indicadores tais como taxa de ocupação e mé dia de permanência para avaliação, ampliação ou redução do número de leitos em determinada especialidade". Anote-se que, em geral, as respostas a estes quesitos, com exceção de alguns entrevistados, pareciam se referir a esforços episódicos, freqüentemente algum levantamento para atender a uma situação "emergencial", não parecendo refletir uma prática planejada e rotineira de uso da informação na gestão.

As respostas ao item "características do sistema de informação" parecem indicar que há pouco investimento nesta área, já que apenas três entre os diretores hospitalares entrevistados declararam que suas instituições são totalmente 
informatizadas, sendo que para os hospitais restantes observou-se até mesmo a presença de coleta manual de dados. Quanto à integração em rede, apenas duas instituições parecem contar com estebenefício.

A grande maioria dos entrevistados afirma que o fluxo de informações é bem definido; porém, a forma concisa e genérica com quefoi respondida esta questão sugere que o investimento nesta área é ainda insuficiente, o que é comprovado pelo fato depoucos hospitais contarem com sistemas informatizados e a minoria desses dispor de rede integrada.

Essa in eficiência da informatização representa um ônus operacional para a instituição, já que os sistemas de registros manuais demandam um elevado custo e grande trabalho de manutenção. Deficiências como registrosilegíveis, multiplicidade de pastas e de critérios de arquivamento, dificuldade de acesso, fragilidade do papel, perdas freqüentes de informações, falta de padronização e dificuldades de efetuar buscas e pesquisas são algumas das questões que poderiam ser minimizadas com o aprimoramento da informatização.

Em referência ao item "mecanismos dedivulgação dos dados e informações", sete dos diretores hospital ares entrevistados declararam a ocorrência de reuniões periódicas com as diretoriase os serviços dos hospitais para discussão e disseminação das informações, sendo que dois deles relataram a ocorrência de reuniões periódicas envolvendo, além da diretoria e dos serviços, a comunidade. Alguns diretores declararam que suas instituições utilizam, como mecanismo de divulgação das informações, um boletim interno encaminhado às clínicas e aos serviços do hospital, mas poucas destas instituições o fazem de forma regular. A penas uma diretoria entrevistada relatou ter como meio de divulgação de informações um mural interno. Quatro diretores hospitalares referiram não possuir quaisquer mecanismos de divulgação de suas informações

No item "perfil da equipe profissional responsável pela análise dos dados e elaboração dos relatórios", os diretores hospitalares apresentaram os seguintes padrões de respostas: "equipe multiprofissional composta por profissionais de nível superior, sendo que todos ou alguns membros da equipe apresentam pós-graduação stricto sensu ou lato sensu"; "equipe multiprofissional, com profissionais de nível médio e superior"; " equipe com coordenação médica sem especialização"; " equipe coordenada por profissionais com formação superior e pós-graduação lato sensu em administração de serviços de saúde ou em saúde pública"; "equipe com coordenação médica especializada em epidemiologia"; e, por último, "equipe não definida". Em geral, as respostas indicaram pouca clareza em relação ao perfil adequado para os profissionais responsáveis pela gestão de informação dentro do hospital.

De um modo geral, a avaliação dos gestores hospitalares sobre o sistema deinformação émais positiva do que a dos responsáveis pela área de informação. Em parte, esse desalinhamento pode ser atribuído ao pequeno envolvimento dos gestores na organização e avaliação regular do sistema de informação, sendo essa condição referida por apenas três dos diretores entrevistados.

A maioria dos diretores hospitalares respondeu afirmativamenteà pergunta sobre a existência de metodologias para conhecer o "perfil da morbidade atendida", sendo citados por ordem decrescentedefreqüência os seguintes instrumentos: relatórios advindos diretamente das clínicas; relatórios do SAM E; relatórios do SAM E associados a outras fontes de dados como o Serviço Social, a AIH ea Fundação Seade; fichas de atendimento; realização de trabal hos científicos; Boletim CAH 106; experiência adquirida e estudo realizado nas clínicas. Também a maior partedos diretores entrevistados relatou exemplos de uso do perfil de morbidade para contribuir na tomada de decisão, citando sua importância para 0 conhecimento da demanda, criação denovos serviços e ampliação dos já existentes, entre outros.

No item "disponibilidade das informações para estudo de tendência", doze dos diretores hospitalares entrevistados referiram dispor de informações obtidas nos anos anteriores. Entre os que afirmam ter informações disponíveis, encontramos desde hospitais que possuem série histórica a partir de 1974, até diretor que relata produzir pessoalmente estas informações apenas para seu próprio uso, sem o envolvimento do SAM E. Embora o Relatório M ensal de Informação Hospitalar tenha sido implantado em 1996 e estar em vigor no momento da pesquisa de campo, alguns entrevistados referiram não existir informações relativas aos anos anteriores para análise de tendência. Frente ao quesito que solicitava "exemplo de estudo de tendência realizado" foram referidos: análise de indicadores, do perfil de morbidade, de produtividade e/ou custos e estudos sobre a demanda.

Em resposta ao item "realização de comparações de seu hospital com outros da administração direta", a maioria dos diretores hospitalares entrevistados relatou não comparar seus resultados aos de outras instituições, sendo que 
apenas sete dos diretores afirmaram avaliar a performance de seu hospital comparando-o a outros da administração direta. Dentre as informações comparadas, foram citadas: número de internações, taxa de ocupação, faturamento, taxa de cesáreas e relação funcionário/leito. Nove diretores comparam os dados dos seus hospitais com outros da administração indireta ou privados/filantrópicos, sendo que a maior parte deles considera os resultados de seus hospitais piores que os dos hospitais privados.

$\mathrm{Na}$ questão sobre "quais seriam os indicadores considerados importantes para apoiar o planejamento e a gestão dos hospitais", os comentários dos diretores seguiram alguns padrões, segundo ordem de freqüência decrescente: indicadores clássicos, tais como média de permanência, taxa de mortalidade, índice de infecção hospitalar, índice de rotatividade eintervalo de substituição; indicadores epidemiológicos; indicadores de custos e administrativos (recursos humanos, materiais efinanceiros) eindicadores de produção e de qualidade. Interessante salientar que quatro dos entrevistados responderam de forma inconclusiva, ou seja, apresentaram dificuldades em elencar indicadores que consideram importantes, denotando pouco conhecimento e habilidade na utilização dos mesmos.

0 resultado das entrevistas pareceindicar que a metodologia de avaliação de desempenho da Secretaria de Saúde, no período estudado, não apresentava mecanismos regulares de feedback para os hospitais com reflexos negativos na motivação dos gestores para a melhoria da qualidade das informações produzidas, como se pode notar na resposta de um dos entrevistados: "temos a sensação de estar enviando uma série de informações sem objetivos claros e concretos".

A quase totalidade dos responsáveis pela área de informações/ SAME relatou que vêm desenvolvendo algumas iniciativas com o intuito de aprimorar seu Sistema de Informação, sendo citados por ordem decrescente defreqüência: "aperfeiçoamento da informatização, melhora dos processos internos e treinamento do pessoal".

\section{Conclusões}

Destaque-se, preliminarmente, que os diretores hospitalares, em sua maioria, parecem ter pouca familiaridade com as propostas relativas à produção e ao uso de informações e que não as incorporam no seu cotidiano de trabalho. Alguns diretores, por exceção, parecem ter uma grande preocupação em solucionar as dificuldades enfrentadas, mas não conseguem, apenas como ato de vontade individual, dar conta dos determinantes institucionais para a mel horia da produção e o uso de informaç̧ões na gestão. Assim, 0 quadro geral traçado a partir das entrevistas revela desconhecimento e restrições, verificandose, no entanto, exceções.

Em geral, os diretores concordam que a seleção dos indicadores não é realizada de acordo com as estratégias dos hospitais e sim com o que ésolicitado pelo nível central da Secretaria deEstado da Saúde, ou, ainda, que simplesmente assumem listas "clássicas" de indicadores. Na percepção desses gestores, não existem mecanismos regulares de feedback dos níveis superiores da SES para os hospitais e acreditam ser este um dos motivos que inviabilizava o processo de comparação entre os hospitais.

Embora boa parte das instituições compare seus indicadores com os de hospitais similares, esta é uma prática informal e episódica. Alguns diretores hospitalares referiram não realizar esta comparação por impossibilidade de acesso às informações de outros hospitais.

Parece existir pouco investimento no sistema de informação hospitalar e, apesar do consenso existentenaliteratura sobrea necessidade de profissionais capacitados para avaliar, em conjunto com os gestores, as informações e os aplicativos deTIC mais eficientes para a realidade do hospital, não se identificou nas entrevistas clareza em relação ao perfil adequado do profissional que trabal ha com gestão de informação dentro dos hospitais.

Os métodos de divul gação de dados e informações nos hospitais estudados revelaram-se insuficientes por referência ao proposto na literatura científica. Entre outras iniciativas, a meIhoria da comunicação entre os gestores e da integração dos sistemas de informação, aliadas a investimentos na informatização, são el ementos fundamentais para alcançar progressos nessa área, sendo essencial o envolvimento dos diretores hospitalares na organização e avaliação regular do sistema de informação.

\section{Considerações finais}

As informações existentes nos hospitais brasileiros quase sempre revelam indicadores clássicos de movimento hospitalar e estes, ainda assim, não são padronizados para os serviços de uma mesma área de abrangência ou entre hospitais 
de mesmo porte. Além disso, os sistemas de informações gerenciais implantados são, habitualmente, pouco valorizados e insuficientemente utilizados pelos níveis gerenciais ${ }^{39}$. Uma evidência desta realidade pode ser encontrada na pesquisa de Malik \& Teles $^{40}$, que mostra apenas $23 \%$ dos hospitais do Estado de São Paulo com iniciativas para a melhoria de qualidade.

Para a maioria das questões analisadas nessa pesquisa, a realidade dos hospitais estudados não parece ter sofrido alterações fundamentais nos últimos anos. Por outro lado, acredita-se que os resultados do presente estudo sejam aplicáveis para a grande maioria dos hospitais brasileiros, tanto os públicos quanto os mantidos por instituições privadas.

\section{Agradecimentos}

Este artigo baseia-se em uma pesquisa iniciada por meio de uma parceria estabelecida entre 0 Programa de Estudos Avançados em Adminis- tração H ospitalar e de Sistemas de Saúde - Proahsa (convênio da FGV-EAESP com o HCFMUSP) e a Secretaria de Estado da Saúde de São Paulo ${ }^{41}$, visando avaliar a qualidade das informações disponíveis e utilização das mesmas na gestão dos hospitais públicos localizados na Região M etropolitana de São Paulo, assi m como sugerir ações para o aprimoramento do Sistema de Informaçõ̃es H ospitalares.

Registra-se aqui 0 agradecimento a todos que colaboraram na elaboração dessa pesquisa, especialmente aos técnicos da SES de São Paulo, professores, funcionários e alunos da Fundação Getulio Vargas - FGV e do PROAH SA. M erecem menção especial Adriana Carro e Aniara Araújo pelo fundamental apoio que ofereceram na fase de redação do relatório da referida pesquisa. Agradecemos também aos diretores dos hospitais e aos responsáveis pelos SAMEs, que concordaram em responder aos questionários e ao GV pesquisa da FGV-EAESP pelo financiamento da pesquisa em que se baseia o presente artigo. 


\section{Referências}

1. Griffith JR, King JG. Championship management for healthcare organizations. J H ealth M anag 2000; 45(1):17-31.

2. NHS UK. A commitment to quality, a quest for excellence: a statement on behalf of the Government, the medical profession and the NHS, NHS, 2001. [acessado 2006 Jun]. Disponível em: www. dh.gov.uk

3. Lemieux-Charles L, Champagne F. Using knowledge and evidence in health care: multidisciplinary perspectives. Toronto: University of Toronto Press; 2004.

4. Fleming ST, Scutchfield FD, Tucker TC. M anagerial epidemiology. Chicago/Washington: AUPHA / HAP; 2000.

5. Novaes HMD. Pesquisa em, sobre e para os serviços de saúde: panorama internacional e questões para a pesquisa em saúde no Brasil. Cad Saúde Pública 2004; 20(Sup 2):S147-S173.

6. Dobrow MJ, Goel V, Upshur REG. Evidence-based health policy: context and utilization. Social Science \& M edicine 2004;58:207-217.

7. Veillard J, et al. A performance assessment framework for hospitals: the WHO regional office for Europe PATH project. Int J for Quality in Health Care 2005; (17)6: 487-496.

8. Noble E, Klein L. Quality assurance: the measure of quality culture in a managed care setting. Total Quality M anagement 2000;11(2):199-205.

9. Turpin RS, et al. A model to assess the usefulness of performance indicators. Int J for Quality in Health Care 1996; 8(4): 321-329.

10. Donabedian A. The epidemiology of quality. Inquiry 1985; 22:282-292.

11. Laudon KC, Laudon JP. Sistemas de informação. Rio de Janeiro: LTC; 1999.

12. Raoufi M. Avoiding information overload: a study on individual's use of communication tools. Proceedings of the 36th Hawaii International Conference on System Sciences 2002. [acessado 2006 Jun]. Disponível em: www.computer.org
13. Curtright JW, et al. Strategic performance management: Development of a Performance M easurement System at the Mayo Clinic. J H ealthc M anag 2000; 45(1): 58-68.

14. Yap C, Siu E, Baker GR, Brown AD. A comparison of systemwide and hospital-specific performance measurement tools. J H ealthc M anag 2005;50(4):251-62.

15. Kaplan RS, Norton DP. A organização orientada para estratégia: como as empresas que adotam a balanced scorecard prosperam no novo ambiente de negócios. Rio de Janeiro: Campus; 2000.

16. Loch $\mathrm{S}$. 0 painel de controle como subsídio à gestão da performance do sistema de saúde: estudo de caso da assistência oncológica em M ato Grosso [dissertação de mestrado]. Florianópolis (SC): Universidade Federal de Santa Catarina; 2002.

17. SM S-SP. Painel de M onitoramento das condições de vida e saúde da população e da situação dos serviços de saúde. [acessado 2006 Jun]. Disponível em: www.prefeitura.sp.gov.br/secretarias/saude

18. Fieschi M, et al. M edical decision support systems: old dilemmas and new paradigms? Tracks for the successful integration and adoption. M eth. Inform. M edicine 2003; 42(3):190-8.

19. Hillestad R, et al. Can electronic medical record systems transform health care? Potential health benefits, savings and costs. Health Affairs 2005; 24(5):1103-1117.

20. Shekelle PG, M orton SC, Keeler EB. Costs and benefits of health information technology. AHRQ Publication 2006; No. 06-E006.

21. M oraes IHS. Política, tecnologia e informação em saúde. Salvador: Casa da Qualidade; 2002.

22. Goman CK. Cinco razões pelas quais as pessoas não dizem o que sabem. [acessado 2006 Jun]. Disponível em: www.kmol.online.pt/artigos

23. Vallet $\mathrm{G}$, et al. Public access to information about the services and quality of care in public hospitals: the need for methodologic clarity: a survey of 44 university hospital directors and administrators. Presse M ed. 2006;35(3pt1):388-92. 
24. Jacobs R, Goddard M, Smith PC. How robust are hospital ranks based on composite performance measures? M ed Care 2005; 43(12):1177-84.

25. Tatchell M. M easuring hospital output: a review of the service mix and case mix approaches. Soc Sci M ed. 1983;17(13):871-883.

26. Lebrão ML. Estudos da morbidade. São Paulo: Editora da Universidade de São Paulo; 1997.

27. Braga Neto $F$, et al. Em busca de novos modelos gerenciais: os Grupos Diagnósticos Homogêneos e a gerência hospitalar. Rev. Adm. Públ. 1990, 24(4): 87-94.

28. Wray NP, et al. The Hospital Multistay rate as an indicator of quality care. Health Services Research 1999; 34(3): 777-790.

29. Noronha M F, Portela M C, Lebrão M L. Potenciais usos dos AP-DRG para discriminar o perfil da assistência de unidades hospitalares. Cad Saúde Pública 2004; 20(Supl 2):S242-S255.

30. Pink GH, Brown AD, Daniel I, et al. Financial benchmarks for Ontario hospitals. Healthc Q 2006; 9(1):40-5.

31. Weiner BJ, Alexander JA, Shortell SM, Baker LC, Becker M, Geppert JJ. Quality improvement implementation and hospital performance on quality indicators. Health Serv Res 2006; 41(2):307-34.

32. Elixhauser A, Pancholi M, Clancy CM. U sing the AHRQ Quality Indicators to improve health care quality. Jt Comm J Qual Patient Saf 2005; 31(9):533-8.

33. Alexander JA, Weiner BJ, Shortell SM, Baker LC, Becker M P. The role of organizational infrastructure in implementation of hospitals' quality improvement. Hosp Top 2006; 84(1):11-20.
34. Hibbard $\mathrm{JH}$, et al. Hospital performance reports: impact on quality, market share, and reputation. Health Aff 2005; 24(4):1150-60.

35. Noronha M.F. Classificação de hospitalizações em Ribeirão Preto: os Diagnosis Related Groups [tese de doutorado]. São Paulo (SP): FSP - USP; 2001.

36. Bittencourt SA, Camacho LAB, Leal M C. O Sistema de Informação Hospitalar e sua aplicação na saúde coletiva. Cad Saúde Pública 2006; 22(1):19-30.

37. Zanetta SFR. M orbidade no Hospital das Clínicas: identificação de perfis e desenvolvimento de instrumento de monitoramento [dissertação de mestrado] São Paulo (SP): FM-USP; 2003.

38. BRASIL. M inistério da Saúde. Portaria no 312 de 02 de Maio de 2002. Brasília 2002.

39. Bittar OJNV. Indicadores de qualidade e quantidade em saúde. RAS 2004; 6(22):15-18.

40. Malik AM, Teles JP. Hospitais e programas de qualidade no Estado de São Paulo. RAE 2001; 41(3) 51-9.

41. Escrivão Junior A. U so de indicadores de saúde na gestão de hospitais públicos da região metropolitana de São Paulo. São Paulo: FGV EAESP; 2004. [Relatório de pesquisa]. Disponível em: www.eaesp.fgvsp.br

Artigo apresentado em 05/10/2006

Aprovado em 24/11/2006

Versão final apresentada em 14/12/2006 